\title{
Laser solid-phase synthesis of single-atom catalysts
}

\author{
Yudong Peng ${ }^{1}$, Jianyun Cao ${ }^{1,2}$, Yang Sha', Wenji Yang ${ }^{1}$, Lin Li ${ }^{3}$ and Zhu Liu' ${ }^{1 凶}$
}

\begin{abstract}
Single-atom catalysts (SACs) with atomically dispersed catalytic sites have shown outstanding catalytic performance in a variety of reactions. However, the development of facile and high-yield techniques for the fabrication of SACs remains challenging. In this paper, we report a laser-induced solid-phase strategy for the synthesis of Pt SACs on graphene support. Simply by rapid laser scanning/irradiation of a freeze-dried electrochemical graphene oxide (EGO) film loaded with chloroplatinic acid $\left(\mathrm{H}_{2} \mathrm{PtCl}_{6}\right)$, we enabled simultaneous pyrolysis of $\mathrm{H}_{2} \mathrm{PtCl}_{6}$ into SACs and reduction/ graphitization of EGO into graphene. The rapid freezing of $\mathrm{EGO}$ hydrogel film infused with $\mathrm{H}_{2} \mathrm{PtCl}_{6}$ solution in liquid nitrogen and the subsequent ice sublimation by freeze-drying were essential to achieve the atomically dispersed Pt. Nanosecond pulsed infrared (IR; $1064 \mathrm{~nm}$ ) and picosecond pulsed ultraviolet (UV; $355 \mathrm{~nm}$ ) lasers were used to investigate the effects of laser wavelength and pulse duration on the SACs formation mechanism. The atomically dispersed Pt on graphene support exhibited a small overpotential of $-42.3 \mathrm{mV}$ at $-10 \mathrm{~mA} \mathrm{~cm}{ }^{-2}$ for hydrogen evolution reaction and a mass activity tenfold higher than that of the commercial Pt/C catalyst. This method is simple, fast and potentially versatile, and scalable for the mass production of SACs.
\end{abstract}

\section{Introduction}

Single-atom catalysts (SACs) are a class of catalysts in which individual and isolated metal atoms are anchored to supports. Due to their maximized atom utilization and unique coordination environments, SACs have emerged as a new frontier in heterogeneous catalysis, showing outstanding catalytic performance in a variety of reactions $^{1,2}$, including electrochemical reactions, water-gas shift reactions, and hydrogenation reactions. The SACs have shown a wide range of applications in synthetic organic chemistry ${ }^{3}$, solar hydrogen technologies ${ }^{4}$, and low-platinum fuel cells ${ }^{5}$.

The production of well-dispersed isolated atoms as heterogeneous catalysts remains challenging due to the high-surface energy of individual atoms. Various strategies have been developed for the fabrication of SACs. High-vacuum physical deposition technologies, such as

\footnotetext{
Correspondence: Zhu Liu (zhu.liu@manchester.ac.uk)

'Department of Materials, School of Natural Sciences, The University of Manchester, Manchester M13 9PL, UK

${ }^{2}$ National Graphene Institute, The University of Manchester, Manchester M13 9PL, UK
}

Full list of author information is available at the end of the article atomic-layer deposition ${ }^{6}$, provide ideal model catalysts for fundamental studies of the metal-support interaction ${ }^{7}$. However, these high-vacuum methods are difficult for large-scale production due to the complex and costly equipment and low yield. Meanwhile, a variety of wetchemical routes, including co-precipitation ${ }^{8}$, impregnation $^{9}$, acid leaching ${ }^{10}$, and de-alloying ${ }^{11}$, are commonly used for catalyst synthesis. The adaptation of these methods in the production of SACs remains limited, for the complexity, poor versatility, long processing time, and high post-processing $\cos ^{12}$. In addition, defect engineering ${ }^{13}$ and organic synthesis (e.g., metal-organic framework $^{14}$ ) also suffer from poor versatility and tedious precursor modifications.

On the other hand, facile fabrication of heterogeneous SACs has been achieved by high-temperature pyrolysis of precursors on a wide variety of supports. For instance, a single atom $\mathrm{Fe}, \mathrm{Co}$, or $\mathrm{Ni}$ on carbon nanotube was obtained by multistep pyrolysis of the mixture of dicyandiamide with iron, cobalt, and nickel acetylacetonate, respectively ${ }^{15}$. Very recently, Yao et al. demonstrated the use of high-temperature shockwaves generated from pulsed electrical Joule heating of the

\section{(c) The Author(s) 2021}

(c) (i) Open Access This article is licensed under a Creative Commons Attribution 4.0 International License, which permits use, sharing, adaptation, distribution and reproduction cc) in any medium or format, as long as you give appropriate credit to the original author(s) and the source, provide a link to the Creative Commons license, and indicate if changes were made. The images or other third party material in this article are included in the article's Creative Commons license, unless indicated otherwise in a credit line to the material. If material is not included in the article's Creative Commons license and your intended use is not permitted by statutory regulation or exceeds the permitted use, you will need to obtain permission directly from the copyright holder. To view a copy of this license, visit http://creativecommons.org/licenses/by/4.0/. 
$\mathrm{H}_{2} \mathrm{PtCl}_{6}$-loaded activated carbon nanofiber to synthesize and stabilize single $\mathrm{Pt}$ atoms ${ }^{16}$. In this research, proper controls of the heating period and temperature $(\sim 1500 \mathrm{~K})$ are critical to avoid agglomeration and provide sufficient activation energy for the formation of a thermodynamically stable $\mathrm{Pt}-\mathrm{C}$ bond. This synthetic method extends the previous high-temperature atom trapping and pyrolysis routes $^{17,18}$, which improves processing efficiency and can be easily scaled up.

Lasers, as a non-contact manufacturing tool, benefit from various available wavelengths, high-energy-density, rapid scanning speed, high spatial resolution, and good flexibility in designing complex patterns and 3D architecture ${ }^{19}$. They have been used to fabricate various kinds of nanoparticles (NPs), including metal, alloy and metal oxide NPs, semiconductor quantum dots, and core-shell $\mathrm{NPs}^{20,21}$, offering a powerful and flexible alternative to the purely chemical approaches for the synthesis of NPs. Laser-induced synthesis of NPs mainly based on two different mechanisms. The first mechanism is based on laser ablation in liquid to produce colloidal NPs with particle size typically above $5 \mathrm{~nm}$ and broad size distribution ${ }^{21}$. The second mechanism is based on laserassisted photochemical processes to generate metal $(\mathrm{Au}$, Ag, and Pd) NPs down to sub- $5 \mathrm{~nm}$ through photochemical reduction of metal salt precursors in aqueous solution, e.g., using ultrashort laser pulses to induce solvated electron from the liquid medium or excite electron-hole pairs in the semiconducting solute that are capable of reducing soluble metal ions to neutral atoms, which then coalesce into $\mathrm{NPs}^{22,23}$. However, the synthesis of atomically dispersed species via liquid-phase laser photo-deposition remains challenging, as the nucleation and crystal growth can hardly be prevented without effective confinement of the reactants.

In this paper, for the first time, we report a laserinduced solid-phase strategy for the synthesis of atomically dispersed Pt on graphene support by simultaneous pyrolysis of metal salt precursor and reduction/graphitization of electrochemical graphene oxide (EGO). The Pt-EGO samples for laser irradiation were prepared by freeze-drying the $\mathrm{H}_{2} \mathrm{PtCl}_{6}$ infused EGO hydrogel films to form "isolated dispersion" of $\mathrm{H}_{2} \mathrm{PtCl}_{6}$ precursor on the EGO substrate. In order to establish laser synthesis mechanisms, two types of lasers, namely, a picosecond (ps) pulsed ultraviolet (UV) laser with a wavelength of $355 \mathrm{~nm}$ and nanosecond (ns) pulsed infrared (IR) Nd:YAG laser with a wavelength of $1064 \mathrm{~nm}$ were used; and the temperature evolution during the laser irradiation was recorded. For the use of the ns IR laser, the localized temperature reached up to ca. $1350.2 \mathrm{~K}$ as measured by the thermal camera; finite element modeling suggested a peak temperature as high as $1692.6 \mathrm{~K}$, suggesting thermal decomposition route via photothermal effect. After the laser irradiation, single Pt atoms decorated laser-reduced EGO (Pt-LrEGO) with a Pt loading of $0.41 \mathrm{wt} . \%$ were successfully synthesized. As electrocatalysts, the PtLrEGO presented a small overpotential of $-42.3 \mathrm{mV}$ at $-10 \mathrm{~mA} \mathrm{~cm}^{-2}$ in hydrogen evolution reaction (HER) with a mass activity over ten times higher than that of the commercial Pt/C. This laser synthesis method is one-step, simple, ultrafast, and potentially scalable, which can be readily extended to other metals and supporting materials for mass production of functional SACs for various applications.

\section{Results and discussion}

The laser synthesis is illustrated in both Fig. 1 and Supplementary Fig. S1. First, the EGO film was prepared by vacuum filtration of the EGO aqueous dispersion through a PTFE membrane filter, then $1 \mathrm{~mL}$ of the chloroplatinic acid $\left(\mathrm{H}_{2} \mathrm{PtCl}_{6}\right)$ solution with desired concentration $(1,2,3,5$, or $10 \mathrm{mM})$ was filtered through the EGO film to obtain the Pt-EGO hydrogel film. The freshly filtered, wet Pt-EGO hydrogel film supported by the PTFE membrane filter was immediately frozen in liquid nitrogen, followed by freeze-drying in a vacuum to sublimate the ice. The purpose of this direct sublimation of solid ice to water vapor was to immobilize the $\left[\mathrm{PtCl}_{6}\right]^{2-}$ anion complex on negatively charged EGO flakes to avoid localized precipitation of the metal precursor. The asprepared Pt-EGO films were subject to laser irradiation under Ar atmosphere via rapid scanning. After the laser treatment, the laser-reduced EGO (LrEGO) film loaded with $\mathrm{Pt}$ single atoms were separated from the PTFE membrane filter by sonication in water, followed by washing and freeze-drying to obtain the final materials in a powder state. The as-formed graphene-supported $\mathrm{Pt}$ SACs are denoted as Pt $x$-LrEGO, where $x$ represents the concentration of the $\mathrm{H}_{2} \mathrm{PtCl}_{6}$ precursor solution filtered through the EGO film, e.g., Pt5-LrEGO represents the concentration of $\mathrm{H}_{2} \mathrm{PtCl}_{6}$ precursor solution is $5 \mathrm{mM}$, and Pt1-LrEGO was prepared by treating the EGO film with $1 \mathrm{mM} \mathrm{H}_{2} \mathrm{PtCl}_{6}$. To distinguish the IR laser-treated LrEGO, the UV-fabricated reduced graphene is denoted as Ptx-LrEGO ${ }_{U V}$.

\section{Characterization of $\mathrm{Pt}$ single atoms}

The samples were characterized with the aberrationcorrected high-angle annular dark-field scanning transmission electron microscope (HAADF-STEM). Figure 2a shows the HAADF-STEM images of the Pt5-LrEGO sample prepared by the ns IR Nd:YAG laser, revealing an intact 2D flake without the presence of Pt nanoparticles or clusters. Nevertheless, Energy-dispersive X-ray (EDX) elemental maps (Fig. 2b) indicate the presence of homogeneously dispersed Pt element over the entire LrEGO support. The HAADF-STEM image at high magnification 

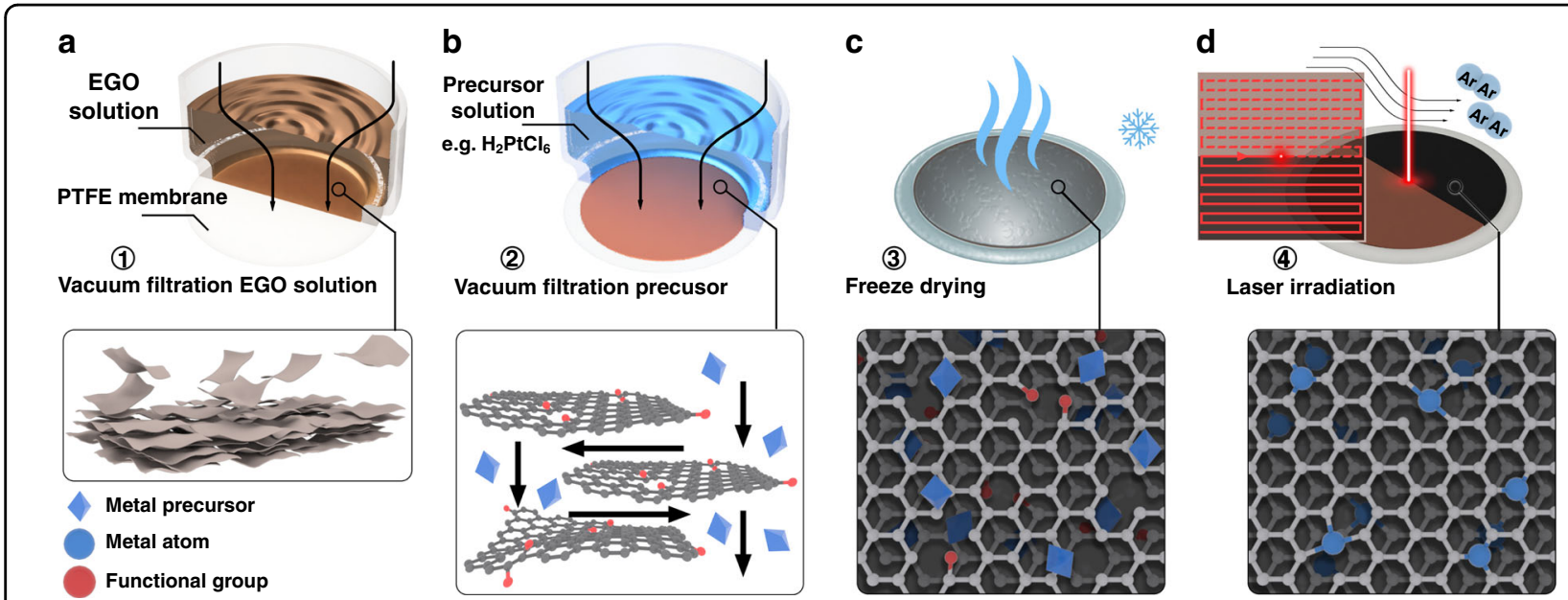

Fig. 1 Schematic illustration of the formation of Pt-LrEGO. a Preparation of EGO film from $1.0 \mathrm{mg} \mathrm{mL}-1 \mathrm{EGO}$ solution through vacuum filtration; b metal precursor filter through the EGO film and followed by (c) immediate freeze in liquid nitrogen and freeze-drying; d direct $1064 \mathrm{~nm}$ laser irradiation of the Pt-EGO to form Pt-LrEGO, inset shows the schematic of the parallel laser scanning path across the EGO film, the laser beam spot size was $1 \mathrm{~mm}$ in diameter and overlap ratio between the tracks was $50 \%$

(Fig. 2c) clearly shows the Pt element on the LrEGO support exists as extremely small bright dots with a diameter of $\sim 0.34 \mathrm{~nm}$, corresponding to the diameter of an individual Pt atom. The HAADF-STEM characterization clearly suggests the presence of single Pt atoms on the EGO support.

In addition to the HAADF-STEM characterization, $\mathrm{X}$-ray diffraction (XRD) and X-ray photoelectron spectroscopy (XPS) further confirm that the Pt species exist in the Pt-LrEGO as uniformly dispersed single atoms. In detail, the XRD pattern of Pt5-LrEGO in Fig. 2d indicates the absence of crystallized Pt nanoparticles, as the characteristic peaks of Pt (111), (200), (220), and (311) (ICDD No. 00-004-0802) that clearly appear in the XRD pattern of commercially available $(\mathrm{CM}) \mathrm{Pt} / \mathrm{C}$ are missing in the XRD pattern recorded from the Pt5-LrEGO sample. XPS Pt $4 \mathrm{f}$ high-resolution spectra provide further evidence for the single atomic dispersion of $\mathrm{Pt}$ in the Pt5-LrEGO sample. As shown in Fig. 2e, the Pt $4 \mathrm{f}$ high-resolution spectra of the $\mathrm{CM} \mathrm{Pt} / \mathrm{C}$ show a set of doublet peaks located at 71.4 and $74.7 \mathrm{eV}$, corresponding to the doublet peaks of $\mathrm{Pt}(0) 4 \mathrm{f}_{7 / 2}$ and $4 f_{5 / 2}$, respectively ${ }^{24}$. For the lasertreated Pt5-LrEGO, the doublet peaks shifted to the higher binding energy of 72.6 and $75.8 \mathrm{eV}$, corresponding to $\mathrm{Pt}(2+)^{24}$. The lack of detectable Pt (0) signal implies that the Pt nanoparticle/clusters were unlikely to exist or with a negligible content in the sample, conforming to the absence of crystallized Pt signals in the XRD patterns. The Pt $(2+)$ originates from the interaction between the single Pt atoms and substrates ${ }^{24}$. The Pt loading for the Pt5-LrEGO is $0.41 \pm 0.01 \mathrm{wt} \%$ as determined by inductively coupled plasma-optical emission spectrometry (ICP-OES).

\section{Effects of precursor concentration and precursor type}

Variation in the concentration of $\mathrm{H}_{2} \mathrm{PtCl}_{6}$ solution filtered through the EGO hydrogel film showed a strong influence on the size of the as-formed Pt species. As shown in Fig. $2 \mathrm{f}$ and $\mathrm{g}$, the laser-treated Pt1-LrEGO using the $1 \mathrm{mM}$ of $\mathrm{H}_{2} \mathrm{PtCl}_{6}$ solution shows atomically dispersive Pt species in a similar size of $\sim 0.34 \mathrm{~nm}$ but with a low Pt loading of $\sim 0.03$ wt.\%. With the increase of the $\mathrm{H}_{2} \mathrm{PtCl}_{6}$ concentration to $5 \mathrm{mM}$, the Pt species remains atomically dispersed on the LrEGO flake (Fig. $2 \mathrm{~h}$ ) while the Pt loading increases to $0.41 \mathrm{wt} . \%$ without noticeable aggregation. Further increasing the concentration of $\mathrm{H}_{2} \mathrm{PtCl}_{6}$ to $10 \mathrm{mM}$ led to clear aggregation of the Pt single atoms (Fig. 2i). The weight percentage of Pt species increased from 0.03 to 1.40 wt.\% with $\mathrm{H}_{2} \mathrm{PtCl}_{6}$ concentration from 1 to $10 \mathrm{mM}$ (Fig. 2f).

In addition to the concentration of precursor solution, the type of Pt precursor compounds, namely, $\mathrm{H}_{2} \mathrm{PtCl}_{6}$ or $\mathrm{PtCl}_{4}$, shows a significant impact on the as-formed $\mathrm{Pt}$ species on the LrEGO support. Unlike the $\mathrm{H}_{2} \mathrm{PtCl}_{6}$ solution that leads to atomically dispersed Pt on the LrEGO flakes, the STEM images (Supplementary Fig. S2) of the Pt-LrEGO synthesized from the $\mathrm{PtCl}_{4}$ precursor via the same laser parameters show prevailing the formation of $\mathrm{Pt}$ nanoparticles for all the concentrations used (from 1 to $10 \mathrm{mM}$ ). We performed XPS analysis for the precursor adsorbed EGO to understand the interfacial interaction between $\mathrm{PtCl}_{4}, \mathrm{H}_{2} \mathrm{PtCl}_{6}$, and the EGO support. Figure 3a presents the XPS survey of the pristine EGO, and EGO infused with $\mathrm{PtCl}_{4}(5 \mathrm{mM})$ and $\mathrm{H}_{2} \mathrm{PtCl}_{6}(5 \mathrm{mM})$ precursors. Except the $\mathrm{C} 1 \mathrm{~s}$ and $\mathrm{O} 1 \mathrm{~s}$ peaks that exist in all samples, the Pt $4 \mathrm{f}$ and $\mathrm{Cl} 2 \mathrm{p}$ peaks were found in both of the precursor infused EGO films. Through comparison of 

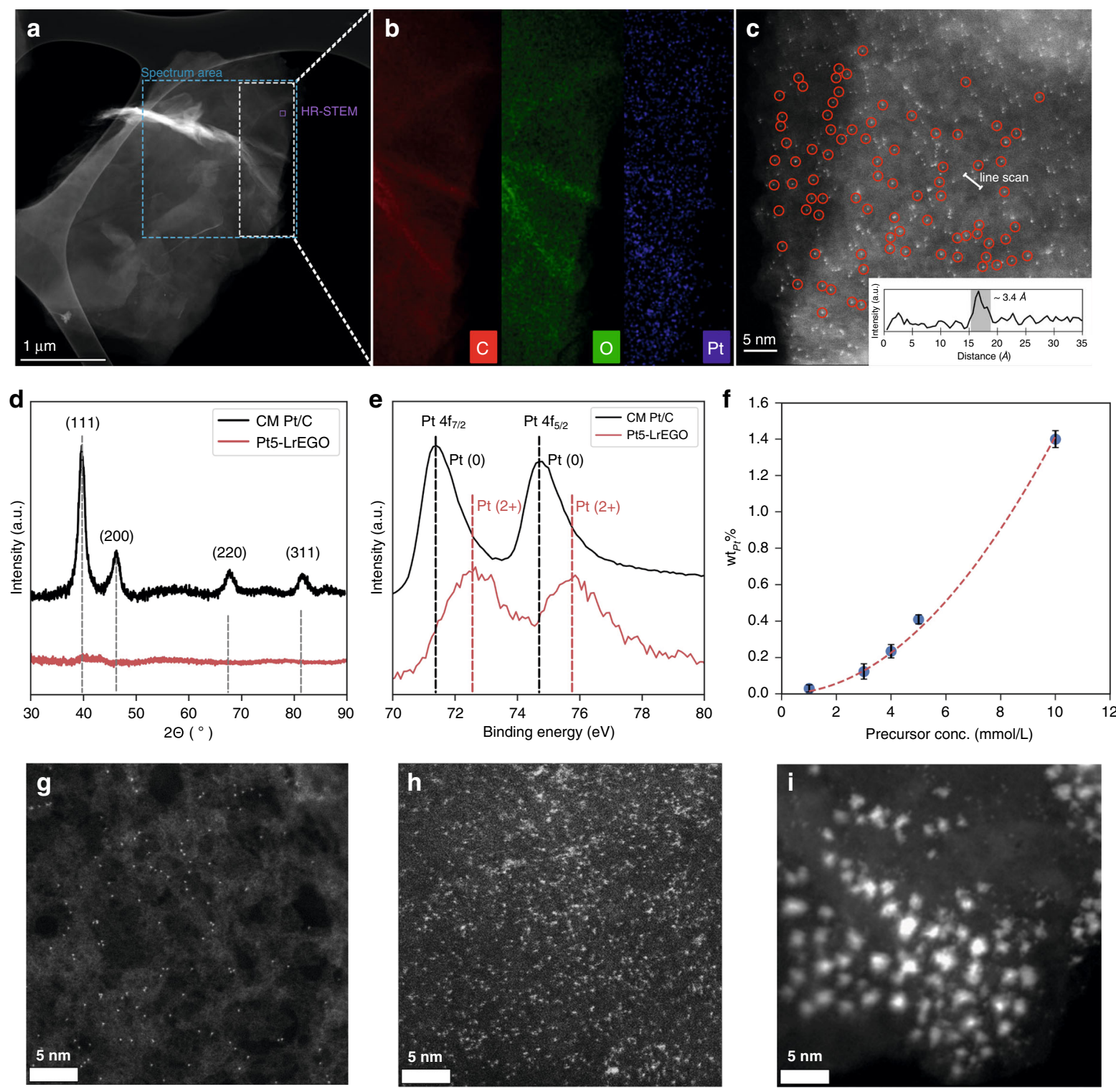

Fig. 2 Structural characterizations of the Pt-LrEGO samples. a HAADF-STEM image of Pt-LrEGO after 1064-nm laser irradiation at a laser fluence of $7.66 \mathrm{~m}^{-2}$ and $\mathbf{b}$ EDX mapping of C, O, and Pt of the selected area. $\mathbf{c}$ Representative HAADF-STEM image showing isolated Pt atoms deposited on LrEGO support (inset shows the representative HAADF intensity profile). $\mathbf{d}$ XRD pattern and e XPS spectra of the commercial Pt and Pt5-LrEGO fabricated by $1064 \mathrm{~nm}$ laser at $7.66 \mathrm{~mJ} \mathrm{~cm}{ }^{-2}$. f ICP-OES result of Pt loading within Pt-LrGO with various precursor concentrations, and representative STEM images of $\mathbf{g}$ Pt1-LrGO, h Pt5-LrGO, and $\mathbf{i}$ Pt10-LrGO processed at $7.66 \mathrm{~mJ} \mathrm{\textrm {cm } ^ { - 2 }}$

the integrated intensity of $\mathrm{Pt}, \mathrm{Cl}, \mathrm{C}$, and $\mathrm{O}$, the percentage of $\mathrm{Pt}$ species in the spectral are $\sim 0.69 \mathrm{at}_{\mathrm{Pt}} \%$ and $\sim 0.06$ $\mathrm{at}_{\mathrm{Pt}} \%$ for $\mathrm{EGO}-\mathrm{PtCl}_{4}$ and $\mathrm{EGO}-\mathrm{H}_{2} \mathrm{PtCl}_{6}$, respectively. The content of adsorbed Pt species on the EGO-PtCl 4 is oneorder magnitude higher than that of the $\mathrm{EGO}-\mathrm{H}_{2} \mathrm{PtCl}_{6}$, clearly indicating the difference in interfacial interaction and adsorption tendency. Besides, in exploring the remaining $\mathrm{Cl}$ element within the samples, we found that the Pt:Cl ratio for $\mathrm{EGO}-\mathrm{H}_{2} \mathrm{PtCl}_{6}$ is $1: 5$, which is close to that of the original compound, while the $\mathrm{Pt}: \mathrm{Cl}$ ratio for EGO-PtCl $l_{4}$ is $\sim 1: 1.5$. Figure $3 \mathrm{~b}$ shows the Pt $4 \mathrm{f}$ spectra of the EGO-PtCl $\mathrm{E}_{4}$ and $\mathrm{EGO}-\mathrm{H}_{2} \mathrm{PtCl}_{6}$, each Pt $4 \mathrm{f}$ peak can be de-convoluted into two pairs of doublets and were assigned to Pt (IV) $\left.\left(\mathrm{PtCl}_{4} / \mathrm{H}_{2} \mathrm{PtCl}\right)_{6}\right)$ Pt (II) $\left(\mathrm{PtCl}_{2}\right)$ and $\mathrm{PtO} / \mathrm{Pt}(\mathrm{OH})_{2}$ (binding energy summarized in Supplementary Table S1) ${ }^{25,26}$. Apart from the Pt (IV) peaks from the precursors, the existence of $\mathrm{PtO} / \mathrm{Pt}(\mathrm{OH})_{2}$ peaks attributes to the hydrolysis of the metal salts by humid air $^{25}$. The dominating Pt (II) components in the Pt $4 \mathrm{f}$ spectrum of $\mathrm{EGO}-\mathrm{PtCl}_{4}$ are possibly caused by the reduction of $\mathrm{Pt}^{4+}$. Owing to the electrophilic nature of Pt (IV), the Pt (IV) can oxidize hydrocarbon and itself 

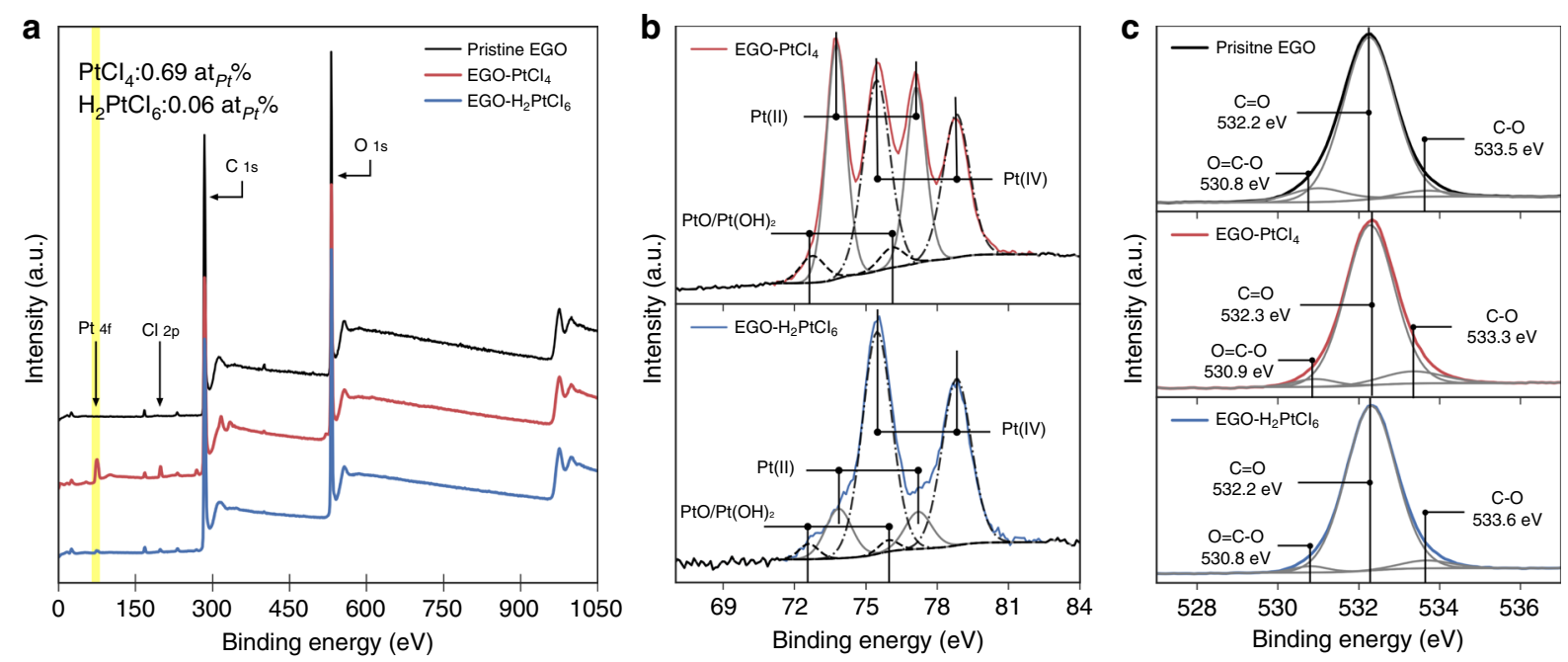

Fig. 3 XPS characterization of the pristine EGO, and $\mathrm{PtCl}_{4}(5 \mathrm{mM})$ and $\mathrm{H}_{2} \mathrm{PtCl}_{6}(5 \mathrm{mM})$ filtered EGO films. a XPS survey, b high-resolution XPS Pt $4 \mathrm{f}$ spectra, and c O $1 \mathrm{~s}$ spectra

turns to $\mathrm{PtCl}_{2}$ and $\mathrm{HCl}^{27}$. In our study, the oxidation of EGO caused by $\mathrm{PtCl}_{4}$ is more pronounced than $\mathrm{H}_{2} \mathrm{PtCl}_{6}$, this explains the disproportion of $\mathrm{Pt}: \mathrm{Cl}$ in the samples. By examining the O1s spectra (Fig. 3c), the integrated area of $\mathrm{C}-\mathrm{O}$ peak of $\mathrm{EGO}-\mathrm{PtCl}_{4}$ among the deconvoluted $\mathrm{O} 1 \mathrm{~s}$ signal is $11.6 \%$, which is two times larger than $5.4 \%$ of EGO- $\mathrm{H}_{2} \mathrm{PtCl}_{6}$. Moreover, the binding energies of the $\mathrm{C}-\mathrm{O}$ group are at 533.5, 533.3, and $533.6 \mathrm{eV}$ for the pristine EGO, EGO-PtCl 4 , and EGO$\mathrm{H}_{2} \mathrm{PtCl}_{6}$, respectively; the relatively smaller binding energy of the $\mathrm{C}-\mathrm{O}$ group in $\mathrm{EGO}-\mathrm{PtCl}_{4}$ provides evidence for the adsorption of Pt species on the epoxy and/ or hydroxyl groups of $\mathrm{EGO}^{26}$.

The excellent hydrophilicity of EGO in water is largely due to the negatively charged oxygen-containing groups on the surface, where hydrated cation tends to interact with the oxidized groups through coupling between the oxygen atoms and the empty orbitals of metal cations ${ }^{28,29}$. The previous research ${ }^{30}$ on quantification of Pt uptake by oxidized carbon substrates indicates the amount of $\mathrm{PtCl}_{6}{ }^{2}$ - taken up was negligible compared to $\mathrm{Pt}\left(\mathrm{NH}_{3}\right)_{2}{ }^{+}$. Similarly, the positively charged $\mathrm{Pt}^{4+}$ from $\mathrm{PtCl}_{4}$ solution is most likely adsorbed on EGO via electrostatic interaction and forms EGO-Pt (II/IV) complex before freeze-drying, which might adversely affect the dispersion of $\mathrm{Pt}^{4+}$ on the EGO substrate, and thus unlikely to form Pt single atoms.

Therefore, the successful formation of single Pt atoms anchored on the LrEGO support in this case strongly relied on controlling the ion distribution on the supporting materials and the precursor concentration and types. Reducing the precipitation of metal precursors and physical increasing the distance of ions/single atoms thus suppress aggregation and formation of clusters/nanoparticles.

\section{Characterization of laser-reduced EGO}

In addition to the formation of single Pt atoms, the quality of the supporting LrEGO also plays an important role in the catalytic performance by building up an electrical conduction network. Supplementary Fig. S3 shows FEG-SEM images of the Pt5-LrEGO prepared using the ns IR laser. A significant expansion of the EGO film was observed in the laser-irradiated area with the film thickness increased $\sim 27$ times from 1.3 to $35 \mu \mathrm{m}$, reflecting a drastic formation and release of gaseous products during the laser irradiation. The XRD patterns of the Pt5-LrEGO irradiated by the ns IR laser (Fig. 4a) show the disappearance of the characteristic diffraction peak of EGO at a $2 \theta=11.9^{\circ}$ for all the laser fluences $\left(0-12.76 \mathrm{~mJ} \mathrm{~cm}^{-2}\right.$, note: zero fluence represents pristine EGO) and the peaks centered at $2 \theta=26.2^{\circ}$, representing (002) planes of restacked graphene, raised gradually with increasing laser fluence, indicating the occurrence of the reduction of EGO. Furthermore, XPS characterization suggests the oxygen contents of EGO dropped from 20.04 to 5.08 at.\%, after laser irradiation. The XPS high-resolution C1s spectra collected from the pristine EGO film and the Pt5-LrEGO films after the IR laser irradiation at $7.66 \mathrm{~mJ}$ $\mathrm{cm}^{-2}$ reveal a significant decrease in oxygen functional groups. The deconvolution of the $\mathrm{C} 1 \mathrm{~s}$ spectrum of the pristine EGO shows four characteristic components, which are assigned to $\mathrm{C} 1 \mathrm{~s}(284.5 \mathrm{eV}), \mathrm{C}-\mathrm{O}(286.4 \mathrm{eV}), \mathrm{C}=\mathrm{O}$ $(287.9 \mathrm{eV})$, and $-\mathrm{COO}-(289.2 \mathrm{eV})$, respectively (details in Supplementary Fig. S4 and Supplementary Table S2) ${ }^{31}$. In comparison, the C1s spectrum of the LrEGO film shows a significant decrease in the proportions of the oxygen components, and the $\pi$ to $\pi^{*}$ peak at $(290.8 \mathrm{eV})$ appears, showing an effective removal of oxygen groups and restoration of the graphitic structure. 

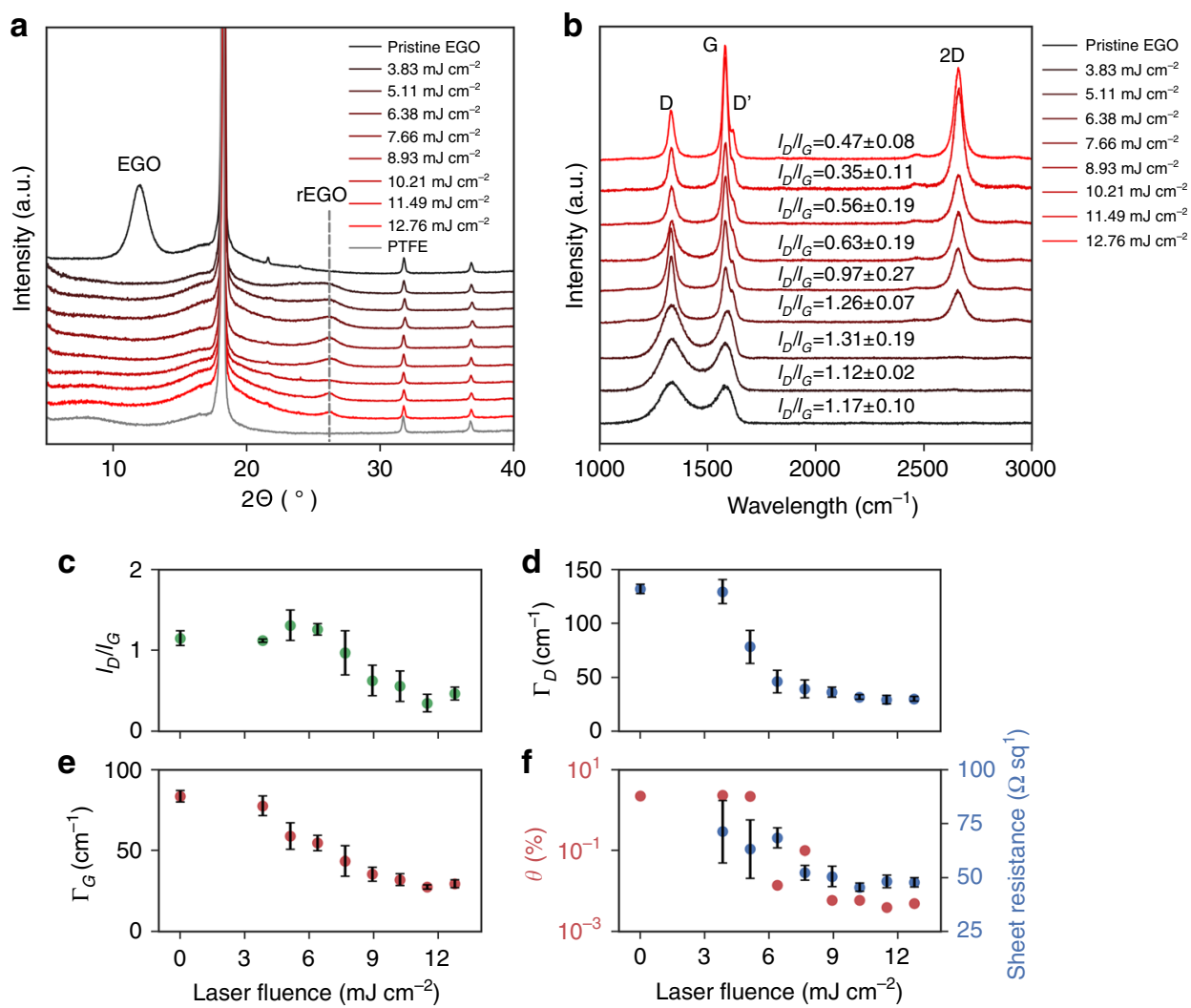

Fig. 4 Characterizations of the Pt-LrEGO prepared with different laser fluences. a XRD patterns (the diffraction peaks centered at $2 \theta=18.1^{\circ}$, $31.7^{\circ}$, and $36.8^{\circ}$ in the XRD pattern originated from the PTFE membrane filter) and $\mathbf{b}$ representative Raman spectra of the pristine EGO and LrEGO after $1064 \mathrm{~nm}$ laser irradiation at various laser fluences. The evolution of $\mathbf{c} / \mathrm{D} / \mathrm{I}_{\mathrm{G}}$ ratio, $\mathbf{d}, \mathbf{e}$ FWHM values of $\mathrm{D}$ and $\mathrm{G}$ bands, and $\mathbf{f}$ defect density $(\theta)$ and sheet resistance of EGO and LrEGO samples

Raman spectroscopy with an excitation wavelength of $633 \mathrm{~nm}$ was used to characterize the graphitization degree of Pt5-LrEGO, the acquired spectra were fitted with Lorentzian function after baseline subtraction for further analysis. Figure $4 \mathrm{~b}$ shows the typical Raman spectra of the pristine EGO and LrEGO samples: the D band located at $\sim 1332 \mathrm{~cm}^{-1}$ represents the edge planes and disordered structures; the $\mathrm{G}$ band at $\sim 1581 \mathrm{~cm}^{-1}$ is induced by the ordered $\mathrm{sp}^{2}$ bonded carbon; the $\mathrm{D}^{c}$ peak centered at $\sim 1619 \mathrm{~cm}^{-1}$ is originated from an intravalley double resonance process; the peak at $\sim 2660 \mathrm{~cm}^{-1}$ is assigned to the $2 \mathrm{D}$ band which is the second-order of the $\mathrm{D}$ peak $^{32,33}$. To monitor the evolution of defect density in the Pt5LrEGO sample after laser irradiation, the $I_{\mathrm{D}} / I_{\mathrm{G}}$ ratio as a function of irradiation fluence was plotted in Fig. 4c. The value of $I_{\mathrm{D}} / I_{\mathrm{G}}$ ratio is closely related to the density of defect/functionality within the graphene basal plane ${ }^{33,34}$. For the use of the ns IR laser as shown in Fig. 4c, the D and $\mathrm{G}$ bands intensity ratio increased initially from $1.17 \pm$ 0.10 to $1.31 \pm 0.19$, followed by a drastic drop to $0.35 \pm$ 0.11 with the increasing laser fluence. This evolution of $I_{\mathrm{D}} / I_{\mathrm{G}}$ ratio fits Ferrari and Robertson's classification of the disorder transition from amorphous carbons to graphite $^{35}$, indicating the decrease of structure disorder with the increase of laser fluence. The reduction and restoration of graphene structure in the Pt5-LrEGO were also supported by the observation of the narrowing of the full width at half-maximum (FWHM: $\Gamma$ ) of D and G bands with the increase of laser fluence (Fig. 4d, e). The model proposed by Cançado et al. ${ }^{33}$ was then applied to quantify the defect distance $\left(L_{\mathrm{D}}\right)$ and defect density $(\theta)$ of the LrEGO films from the Raman $I_{\mathrm{D}} / I_{\mathrm{G}}$ ratios (Supplementary Table S3). Figure 4f shows the defect density retained $\sim 2.25 \%$ at the laser fluence of $6.38 \mathrm{~mJ} \mathrm{~cm}^{-2}$, and then dropped precipitously and down to $\sim 0.05 \%$ at a laser fluence of $7.66 \mathrm{~mJ} \mathrm{~cm}^{-2}$. This observation provides clear evidence for the efficient removal of oxygen groups along with the restoration of the graphene structure under the ns IR laser irradiation. The probe of the sheet resistance change after laser irradiation provides further evidence for the restoration of the electrically conductive $\mathrm{sp}^{2}$ structure. The sheet resistance of the Pt5-LrEGO film (Fig. 4f) decreases with the laser fluence from 3.83 to $6.38 \mathrm{~mJ} \mathrm{~cm}^{-2}$ and remains nearly constant at higher fluence up to 
$12.76 \mathrm{~mJ} \mathrm{~cm}^{-2}$. A minimum sheet resistance value of $45.6 \Omega \mathrm{sq}^{-1}$ was achieved at the laser fluence of $10.21 \mathrm{~mJ}$ $\mathrm{cm}^{-2}$. Note the pristine EGO is an insulator and the resistance value is beyond the instrument detection limit.

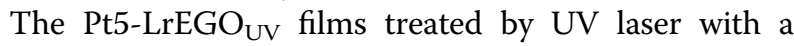
relatively narrower fluence range and denser pulse overlap conditions compared with the IR laser also show a similar trend in $I_{\mathrm{D}} / I_{\mathrm{G}}$ ratio, $\Gamma_{\mathrm{D}}$ and $\Gamma_{\mathrm{G}}$ values with the increase of laser fluence (Supplementary Fig. S5). The ordered crystalline structure occurred in a fluence range from 1.8 to $2.0 \mathrm{~mJ} \mathrm{~cm}^{-2}$. However, at a laser fluence below $1.8 \mathrm{~mJ}$ $\mathrm{cm}^{-2}$, the relatively high $I_{\mathrm{D}} / I_{\mathrm{G}}$ ratio and $\Gamma_{\mathrm{D}}$ and $\Gamma_{\mathrm{G}}$ values, together with the broadened standard deviation (Supplementary Table S4, statistical Raman analysis was based on 10 spectra recorded at random points of each sample), indicate the reduction degree of EGO is low and uneven. The poor reduction degree is also reflected by the sheet resistance of Pt5-LrEGO ${ }_{U V}$. Supplementary Fig. S5e shows that the Pt5-LrEGO tances at increasing laser fluence, but with much less extent than that seen in the IR-irradiated LrEGO. The unevenness in reduction degree was further verified by XRD (Supplementary Fig. S6). The Pt5-LrEGO UV films treated by the ps UV laser show a clear peak at $2 \theta=11.9^{\circ}$, and the peak intensity gradually decreased and completely disappeared when the laser fluence reached $2.0 \mathrm{~mJ} \mathrm{~cm}^{-2}$, while the graphite (002) peaks increased progressively at the laser fluence exceeding $1.45 \mathrm{~mJ} \mathrm{~cm}^{-2}$. When the laser

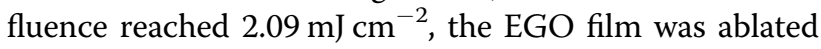
completely from the PTFE membrane.

Taken together, the Raman, XRD, and sheet resistance results strongly suggest the photoreduction of EGO through IR and UV radiation occurs in different photoinitiated manners. From a practical perspective, efficient and even reduction of EGO film was found in IR irradiation, which is evidenced by the high crystallinity and low sheet resistance.

\section{SACs laser synthesis mechanisms}

The laser synthesis introduced in this work was based on photoreduction of the metal precursor and EGO simultaneously. Unlike other chemical and thermal reduction methods, laser reduction is characterized by energy absorption by the GO films dependent on the radiation wavelength and pulse width. From the wavelength perspective, the photoreduction of GO generally can be achieved photochemically with radiation wavelength in the UV region ${ }^{36}$ and photothermally using radiation from visible to $\mathrm{IR}^{37}$. From the temporal perspective, the laser-matter interaction occurs in a clear time sequence ${ }^{38}$ : excitation of an electron by incident photon occurs in the first $\sim 100 \mathrm{fs}$, following by electron-phonon coupling from $100 \mathrm{fs}$ to $10 \mathrm{ps}$, finally from $100 \mathrm{ps}$ or longer heat dissipate to the surrounding matrix. Therefore, thermal analysis is invalid for fs laser irradiation. In our current study, the pulse width of $5 \mathrm{~ns}$ is sufficient for heat conducting to the bulk materials.

When the ns IR laser with a photon energy of $1.17 \mathrm{eV}$ irradiated on the EGO surface, the absorbed laser energy was rapidly converted into local heat via photothermal effect and raise the local temperature, depending on the laser-processing conditions i.e., laser fluence and number of pulses per point (NOPs) determined by the beam size, scanning speed, and repetition rate. To evaluate the temperature profile of EGO film under laser irradiation, we conducted thermal camera measurement of EGO to record the temperature images of EGO scanned by $1064 \mathrm{~nm}$ laser at various fluences. Figure 5 a shows the thermal images of the EGO surface captured at different stages of the laser scanning process at the laser fluence of $7.66 \mathrm{~mJ} \mathrm{~cm}{ }^{-2}$. To compare the temperature evolution of the EGO surface at different laser fluences, we monitored the temperature evolution at five probe points (as marked in Fig. 5a) for each laser fluence. By averaging the time trace temperatures of these five probe points (see details in Supplementary Fig. S7), we obtained the representative temperature-time profiles for each laser fluence. Figure $5 \mathrm{~b}$ shows the temperature evolution of the EGO surface at the laser fluences of $3.83,7.66$, and $11.49 \mathrm{~mJ} \mathrm{~cm}^{-2}$. Figure $5 \mathrm{c}$ compares the peak temperatures at various laser fluences under the same NOP (i.e., 30). It is obvious that the increase of the laser fluences results in the increase of temperatures up to $1350.2 \pm 50 \mathrm{~K}$ at the laser fluence of $11.49 \mathrm{~mJ} \mathrm{~cm}^{-2}$. In addition, due to the high repetition rate $(30 \mathrm{kHz})$, the heat spike induced by each pulse was not able to cool down to the initial temperature between pulses. Therefore, the heat accumulation results in surface temperatures being over $493.2 \mathrm{~K}$ for a period up to $\sim 150 \mathrm{~ms}$. However, owing to the fast temperature change and limited frame rate of the infrared camera, the observed time trace temperature profiles was a result of heat accumulation rather than the actual heating pattern.

In order to reveal the thermal evolution, COMSOL Multiphysics software was used to simulate the EGO film temperature dynamics undergoing $1064 \mathrm{~nm}$ laser irradiation at varying laser fluences. Owing to the extremely small duty cycle of $1 / 6660(5 \mathrm{~ns}, 30 \mathrm{kHz})$, the calculation of the entire process requires tremendous computing time. Thus, the simulation was conducted by depositing 30 successive pulses scanned across the surface probe at $1000 \mathrm{~mm} \mathrm{~s}^{-1}$ with an interval of $\sim 33.3 \mu$ s (Fig. 5d). Each individual pulse possesses a Gaussian energy distribution and has a laser ON time of $5 \mathrm{~ns}$. The simulated temporal thermal profile shown in Fig. 5e shows a short heating time of $\sim 1 \mathrm{~ms}$ to reach up to $1692.2 \mathrm{~K}$, and a fast thermal relaxation to $298.15 \mathrm{~K}$ within $\sim 108 \mathrm{~ms}$. At a given laser fluence, the rapid heating and cooling during and after the laser ON period were clearly shown, and the peak 
a
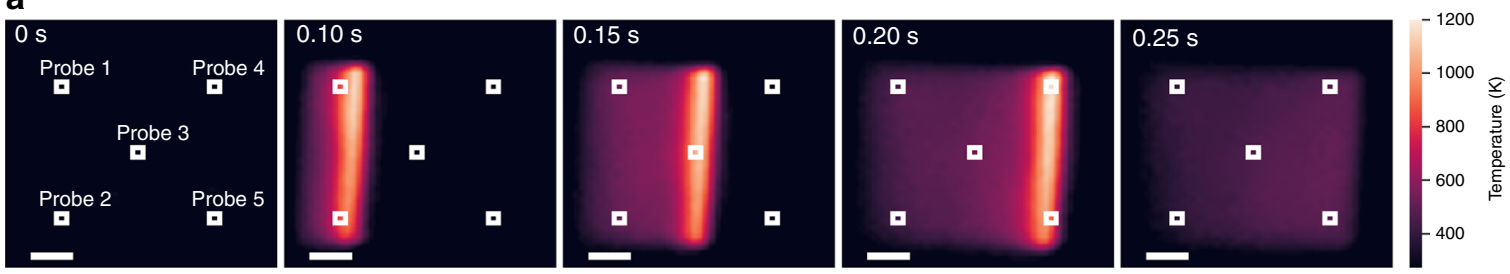

b

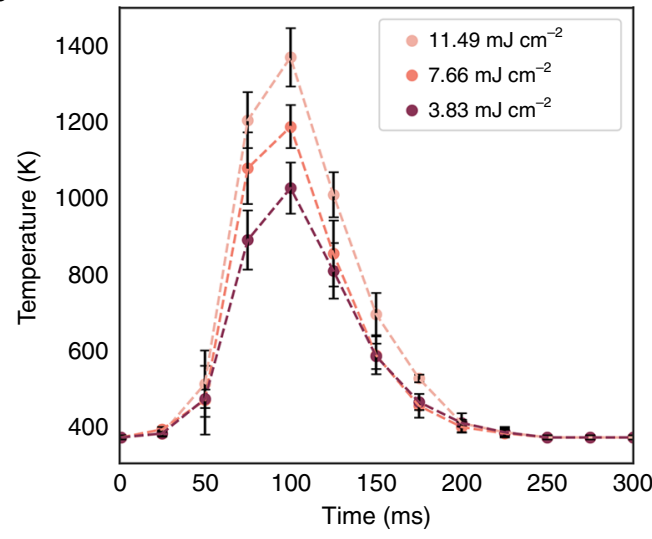

d
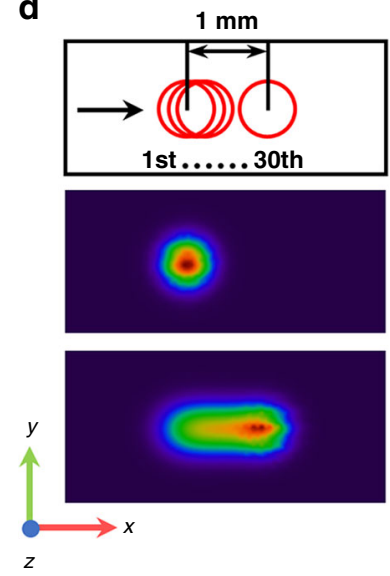

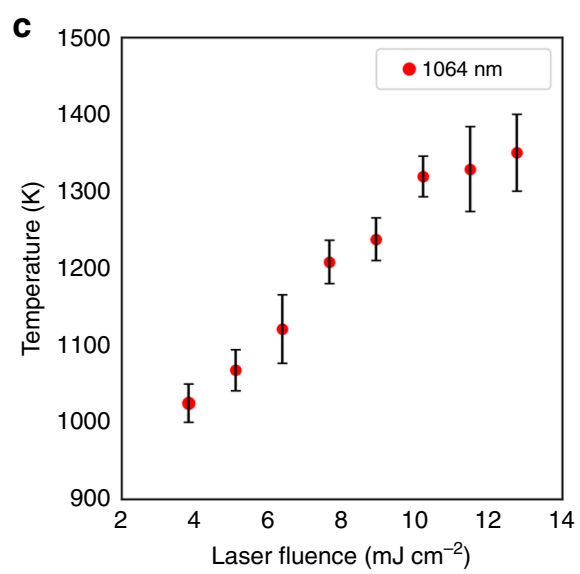

e

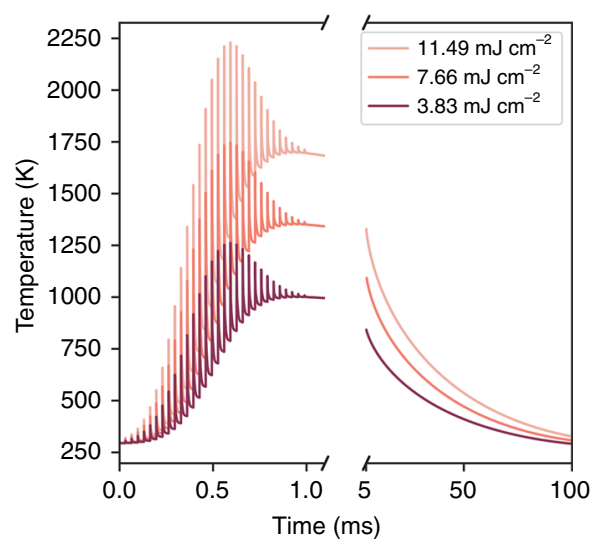

Fig. 5 Thermal profiles of the EGO films under irradiation with different laser fluences. a The infrared images of EGO under $1064 \mathrm{~nm}$ laser irradiation at an average power of $6 \mathrm{~W}$ and at a scanning speed of $1000 \mathrm{~mm} \mathrm{~s}^{-1}$ (the scale is $2.5 \mathrm{~mm}$ ); $\mathbf{b}$ typical temporal thermal profiles at an incident laser fluence of $3.83,7.66$, and $11.49 \mathrm{~m} \mathrm{~cm}^{-2}$, respectively. $\mathbf{c}$ The change of the measured peak temperatures with the variation of laser fluences under at a scanning speed of $1000 \mathrm{~mm} \mathrm{~s}^{-1}$. $\mathbf{d}$ Schematic of 30 successive laser pulse train irradiated on the EGO surface in COMSOL modeling, and the temperature fields on the top surface of EGO film after the first and the thirtieth pulse irradiation. e COMSOL modeling of the EGO film processed by $1064 \mathrm{~nm}$ laser at various laser fluences

temperature gradually rises with increasing accumulated heat and spatial energy density. When the laser beam bypassed the probe, the accumulated heat reached the highest value of 998.9, 1348.2, and $1692.6 \mathrm{~K}$ which corresponds to the laser fluences of $3.83,7.66$, and $11.49 \mathrm{~mJ}$ $\mathrm{cm}^{-2}$, respectively.

As described in Schweizer and Kerr's work ${ }^{39}$, the thermal breakdown of $\mathrm{H}_{2} \mathrm{PtCl}_{6}$ to form platinum metal takes place through several steps as follows: $\mathrm{H}_{2} \mathrm{PtCl}_{6} \rightarrow$ $\mathrm{PtCl}_{4}+2 \mathrm{HCl}$ at $423.2-453.2 \mathrm{~K}, \mathrm{PtCl}_{4} \rightarrow \mathrm{PtCl}_{2}+\mathrm{Cl}_{2}$ at
$574.2-594.2 \mathrm{~K}, \mathrm{PtCl}_{2} \rightarrow \mathrm{Pt}+\mathrm{Cl}_{2}$ at $648.2-783.2 \mathrm{~K}$. Therefore, the temperature of $1025 \mathrm{~K}$ achieved during the $1064 \mathrm{~nm}$ laser irradiation at the lowest laser fluence $\left(3.83 \mathrm{~mJ} \mathrm{~cm}^{-2}\right)$ was sufficient to induce in situ thermal decomposition of the adsorbed $\mathrm{H}_{2} \mathrm{PtCl}_{6}$ molecules in the EGO film to form Pt single atoms. The rapid heating and cooling during the laser processing inhibit the diffusion and aggregation of the $\mathrm{Pt}$ atoms, and the short thermal pulse at temperatures up to $1692.2 \mathrm{~K}$ is likely to promote the formation of stable bonding between the Pt single 
atoms and the graphene support. Interestingly, at the $\mathrm{H}_{2} \mathrm{PtCl}_{6}$ concentration of $5 \mathrm{mM}$, the as-formed Pt species remain as atomically dispersed single atoms with the increase of laser fluence from 3.83 to $11.49 \mathrm{~mJ} \mathrm{~cm}^{-2}$ (Supplementary Fig. S8), indicating that the rise in temperature had not promoted the diffusion of the single atoms, which suggested stable metal-substrate bonding and high stability of the atomic dispersion.

This can be further supported by the consideration of two types of overlapping involved in the beam scanning. One is that as described in Fig. 1, the laser beam spot size was $1 \mathrm{~mm}$ in diameter and overlap ratio between the tracks was $50 \%$. This means that $50 \%$ of the laser track with already formed Pt single atoms was re-treated and a portion adjacent to the successive track was re-heated. However, no Pt aggregation/clusters were evident. The other type of overlapping is always present in pulsed-laser scanning. For instance, with the beam size of $1 \mathrm{~mm}$, the scanning speed of $1000 \mathrm{~mm} / \mathrm{s}$ and repetition rate of $30 \mathrm{kHz}$, the number of pulses per point (NOPs) is 30, which means that the Pt single atoms were exposed to the successive pulses, but no Pt aggregation was observed. This further indicated the stability of the Pt single atoms once synthesized and anchored to the supporting material was formed. On the other hand, thermal reduction of EGO involves the removal of functional groups and restoration of carbon structures. Although the reduction of carboxyl groups with low stability starts at $100-150{ }^{\circ} \mathrm{C}^{40}$, total elimination of all the functional groups including highly stable carbonyl groups needs a high temperature $\left(>1000^{\circ} \mathrm{C}\right)^{41}$, and carbon structural evolution is temperature-dependent. During the laser irradiation, the temperature range achieved was sufficient to induce the photothermal reduction of the EGO, and the re-heating caused by overlapping should be beneficial to the reduction degree.

In order to investigate the effect of the laser irritation time, the scanning speed was set to be $800 \mathrm{~mm} / \mathrm{s}$ with the same laser fluence to $7.66 \mathrm{~mJ} \mathrm{~cm}^{-2}$ to avoid damage of the EGO layer. In this case, the Pt particles/clusters were found in Supplementary Fig. S9, implying that the duration, which also enhances the degree of overlapping, played an important role on atomic diffusion/aggregation of Pt single atoms.

When the ps UV laser was applied, photochemical removal of functional groups was expected ${ }^{42}$. The photon energy of the $355 \mathrm{~nm}$ light is $3.49 \mathrm{eV}$ can excite singlephoton-mediated valence-to-conduction band transition of EGO (with a direct bandgap range from 3.25 to $3.95 \mathrm{eV})^{36,43}$. While for the $1064 \mathrm{~nm}$ beam at a photon energy of $\sim 1.17 \mathrm{eV}$, multiphoton process is required to excite EGO. However, the peak temperature raises of the EGO surface monitored by the thermal camera (Supplementary Fig. S10) reached $803.7 \mathrm{~K}$ at the laser fluence of
$2.0 \mathrm{~mJ} \mathrm{~cm}^{-2}$ and the number of pulses of $\sim 404$, suggesting that both photochemical and photothermal heating took place simultaneously. Since the UV laser $(355 \mathrm{~nm}$, $\sim 4.86 \mathrm{eV}$ ) photon energy is far below the resonant excitation of $\mathrm{C}-\mathrm{O}$ or $\mathrm{C}=\mathrm{O}$ bond ${ }^{22}$, direct excitation may occur through valence-to-conduction band transitions, which may lead to ejection of plasma in the material ${ }^{43}$ and/or photoreduction of EGO film by photon excited electrons of residual water molecules ${ }^{22}$ or EGO itself $f^{36}$. As the direct ionization occurs ahead of thermal accumulation $^{43,44}$, the photoreduction of the EGO could occur via combined mechanisms with the following sub-processes: (i) removal of oxygen-containing functional groups, (ii) conversion of reduced carbon $\mathrm{sp}^{3}$ into $\mathrm{sp}^{2}$ structure, and (iii) restoration of $\mathrm{sp}^{2}$ network. The removal of functional groups and the conversion of $\mathrm{sp}^{2}$ structure can be achieved by both photochemical and photothermal routes, but the restoration of $\mathrm{sp}^{2}$ network might be dominated by thermal effect ${ }^{45,46}$. As demonstrated in Supplementary Figs. S5e and 4f, the defect density of the LrEGO by the ps UV laser was higher than that by the ns IR laser, indicating the higher temperature in the ns IR laser irradiation might benefit the restoration of $\mathrm{sp}^{2}$ carbon network.

In addition, the EGO films contain multiple layers of EGO flakes, one of the reasons of the nonuniform reduction of EGO under UV laser irradiation might be from anisotropy of thermal conductivity in twodimensional materials; and GO has over 100-fold anisotropy of heat flow between the in-plane and out-of-plane directions ${ }^{47}$. For an ultrashort pulsed laser in the ps or fs region, thermal diffusion volume is smaller than that of an ns laser, which results in a superficial reduction of EGO film with poor restoration of $\mathrm{sp}^{2}$ carbon $^{42}$. Furthermore, laser beam conversion into thermal energy depends on not only the wavelength, pulse width, but also the stacked layers ${ }^{48}$. The penetration depth of the UV laser is smaller than that of the IR laser, and the ns pulse width also enhances heat conduction deeper than the ps pulse width. Thus, a thoroughly thermal reduction of EGO film can be expected when using ns IR irradiation. This was reflected by the XRD patterns (Supplementary Fig. S6) that the signals from the EGO were still present for the ps UVtreated Pt5-LrEGO, particularly for the low end of the laser fluences. This was also supported by the observation of the large error bars from ten measurements for various Raman parameters (Supplementary Fig. S5), suggesting that less uniform and smaller penetration of heating by the ps UV laser. Therefore, despite the effective removal of oxygen via photochemical dominating mechanism by the UV laser, reduction and structure restoration of the beneath layers are most likely achieved by thermal conduction with limited heat transport. Increasing the laser fluence of the ps UV laser caused ablation of EGO which 
accompanies endothermic phase changes and further impedes heat conduction. It could be concluded that the ns IR laser irradiation promotes an effective and uniform reduction of EGO throughout the EGO film in the thickness of $1.3 \mu \mathrm{m}$ via a photothermal route.

In comparison to other chemical and physical methods in the fabrication of SACs, the laser photoreduction method is a one-step process, ultrafast laser manufacturing technique for the fabrication of Pt single atoms on the LrEGO support. Since the processing temperature by the ns IR laser can readily reach over $1350.2 \mathrm{~K}$, which is sufficient to thermally decompose most of the metal precursors.

\section{Electrocatalytic performance}

The electrocatalytic HER activities of the Pt-LrEGO samples treated by the ns IR laser was evaluated using a typical three-electrode system with a rotating disk electrode (RDE). The measurement was conducted in a $\mathrm{N}_{2}$ saturated $0.5 \mathrm{M} \mathrm{H}_{2} \mathrm{SO}_{4}$ electrolyte at a rotating speed of $1600 \mathrm{rpm}$. To reveal the intrinsic catalytic performance, the ohmic-drop correction was conducted in order to minimize the solution resistance. The linear sweep voltammogram (LSV) curves of the purchased commercial Pt/C (20 wt.\%; Johnson Matthew, Highspec3000), LrEGO and Pt-LrEGO samples with varying Pt loading are shown in Fig. 6a. Note that the Pt1-LrEGO and Pt5-LrEGO have Pt species in the form of single atoms, while the Pt species in the Pt10-LrEGO present as clusters. The results show that the incorporation of Pt into LrEGO in a form of either single atoms or clusters enhances its HER activity, as the pure LrEGO exhibits negligible current change under the applied voltage of $-0.3 \mathrm{~V}$ vs RHE. Using the thermodynamic HER potential $\left(\mathrm{H}^{+} / \mathrm{H}_{2}=0 \mathrm{~V}\right.$ vs RHE) as a reference, the Pt10-LrEGO and Pt5-LrEGO exhibited small overpotentials of 40.2 and $42.2 \mathrm{mV}$ to drive a cathodic current density of $10 \mathrm{~mA} \mathrm{~cm}^{-2}$, respectively, which are comparable to that of the commercial $\mathrm{Pt} / \mathrm{C}$ catalyst $(32.2 \mathrm{mV})$. With the further decrease of Pt loading to $\sim 0.03 \%$, the Pt1-LrEGO shows a large HER overpotential of $157.8 \mathrm{mV}$ at $10 \mathrm{~mA} \mathrm{~cm}^{-2}$, indicating the lack of active sites on the LrEGO support.

Electrochemical impedance spectroscopy (EIS) was performed at an overpotential of $30 \mathrm{mV}$ to evaluate the electrode kinetics for HER (Supplementary Fig. S11) ${ }^{6,49}$. The Nyquist plot indicates the Pt5-LrEGO possesses a low charge-transfer resistance $\left(R_{\mathrm{ct}}\right)$ of $9.88 \Omega$ implying a fast Faradaic process between the catalysts and electrolyte has been achieved. The HER catalysis kinetics of all samples were assessed by Tafel plot, as shown in Fig. 6b. The resultant Tafel slope of Pt10-LrEGO $\left(31.8 \mathrm{mV} \mathrm{dec}^{-1}\right)$ and Pt5-LrEGO (33.8 $\mathrm{mV} \mathrm{dec}^{-1}$ ) are comparable to the CM Pt/ $\mathrm{C}\left(32.3 \mathrm{mV} \mathrm{dec}^{-1}\right)$, signifying superior HER kinetics in spite of the extremely low Pt loading (1.4 $\mathrm{wt}_{\mathrm{Pt}} . \%$ and $0.41 \mathrm{wt}_{\mathrm{Pt}} . \%$ vs $20 \mathrm{wt}_{\mathrm{Pt}} \%$ ). As expected, the Pt1-LrEGO shows a large Tafel slope of $92.2 \mathrm{mV} \mathrm{dec}^{-1}$ due to the lack of active sites.

The mass activities of the catalysts were evaluated by normalizing the measured current at a given overpotential to the Pt mass loading, and an overpotential of $50 \mathrm{mV}$ was chosen to compare with the literature reported values. As shown in Fig. 2f, because of the significantly lower Pt loading in the Pt5-LrEGO $\left(0.41 \mathrm{wt}_{\mathrm{Pt}} \%\right.$ ) compared to the $\mathrm{CM} \mathrm{Pt} / \mathrm{C}$ (20 $\mathrm{wt}_{\mathrm{Pt}} \%$ ), the Pt5-LrEGO shows an order of

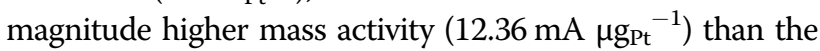
$\mathrm{CM} \mathrm{Pt} / \mathrm{C}\left(0.83 \mathrm{~mA} \mu \mathrm{g}_{\mathrm{Pt}}{ }^{-1}\right)$. This superior HER mass activity of Pt5-LrEGO outperforms the majority of literature values (Fig. 6c, a detailed comparison is given in Supplementary Table S6), demonstrating ultrahigh Pt utilization and great promise for practical applications. Compared to the Pt5LrEGO, the Pt10-LrEGO shows a moderate enhancement in mass activity, which is likely because of a fraction of inert Pt atoms embedded inside the clusters.

We subsequently studied the influence of laser fluence on the HER performance of the Pt single atoms decorated LrEGO. The Pt5-LrEGO treated by the $\mathrm{ns} 1064 \mathrm{~nm}$ laser at 3.83, 7.66, and $11.49 \mathrm{~mJ} \mathrm{~cm}^{-2}$ are denoted as Pt5LrEGO-3.83, Pt5-LrEGO-7.66, and Pt5-LrEGO-11.49, respectively, and sample fabricated via ps $355 \mathrm{~nm}$ laser-irradiated at $2.00 \mathrm{~mJ} \mathrm{~cm}{ }^{-2}$ sample is named as Pt5$\mathrm{LrEGO}_{\mathrm{UV}}$. Figure $6 \mathrm{~d}$ shows the LSVs of above-mentioned catalysts tested in $0.5 \mathrm{M} \mathrm{H}_{2} \mathrm{SO}_{4}$ electrolyte, the overpotential at $10 \mathrm{~mA} \mathrm{~cm}^{-2}$ of Pt5-LrEGO-3.83, Pt5-LrEGO7.66, and Pt5-LrEGO-11.49 were 43.3, 42.2, and $43.1 \mathrm{mV}$, respectively, demonstrating the HER activity and kinetics remain unchanged with various laser outputs under this fabrication condition. The Pt5-LrEGO ${ }_{U V}$ sample shows a slightly increased overpotential of $52.6 \mathrm{mV}$ at $10 \mathrm{~mA}$ $\mathrm{cm}^{-2}$, possibly owing to the unevenly reduced LrEGO, which leads to a relatively larger charge-transfer resistance $\left(R_{\mathrm{ct}}=14.2 \Omega\right.$ at an overpotential of $30 \mathrm{mV}$, Supplementary Fig. S11). Chronopotentiometric test at $10 \mathrm{~mA} \mathrm{~cm}^{-2}$ has been performed to evaluate the HER durability of Pt5-LrEGO samples. As shown in Fig. 6e, the Pt5-LrEGO-11.49 maintains a stable HER performance with a slight increase of overpotential by $39 \mathrm{mV}$ after the $10 \mathrm{~h}$ testing. Nevertheless, the HER activity of Pt5LrEGO-7.66 and Pt5-LrEGO-3.83 degrade remarkably after $10 \mathrm{~h}$ with a final potential of -0.1 and $-0.13 \mathrm{~V}$ vs RHE to drive $10 \mathrm{~mA} \mathrm{~cm}^{-2}$ current density, indicating the Pt5-LrEGO HER durability increased with increasing laser fluence. STEM images acquired from the Pt5-LrEGO samples after the durability test are shown in Supplementary Fig. S12. By comparing Supplementary Fig. S8c and Supplementary Fig. S12a, b, there is only a slight increase in Pt size due to aggregation for the Pt5-LrEGO11.49 after the durability test. This result implies a better durability of Pt5-LrEGO-11.49 SAC in the HER. In contrast, the Pt atoms coalesced into clusters and larger 

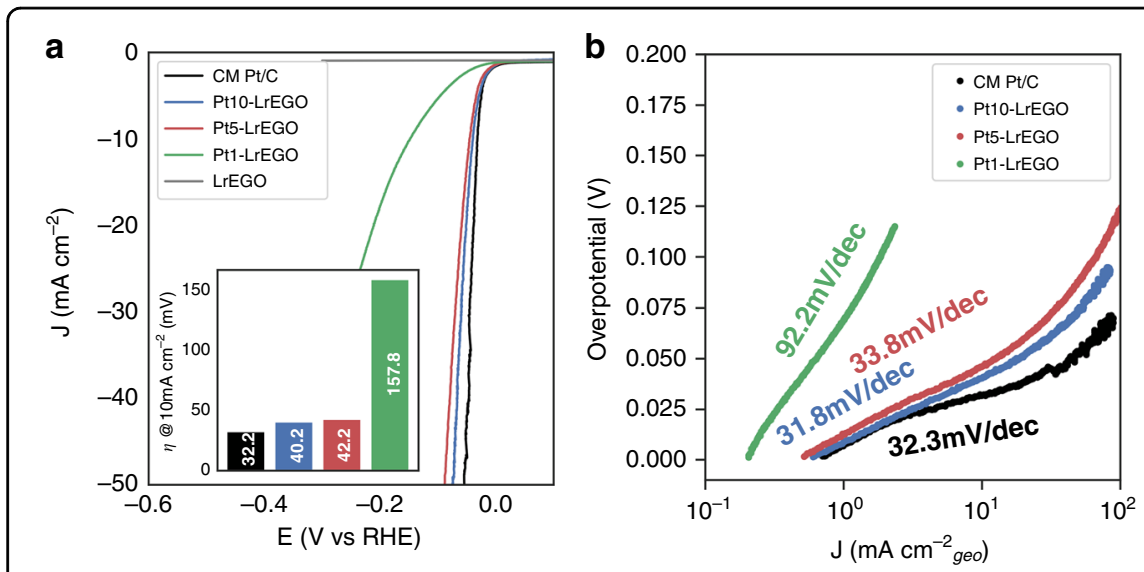

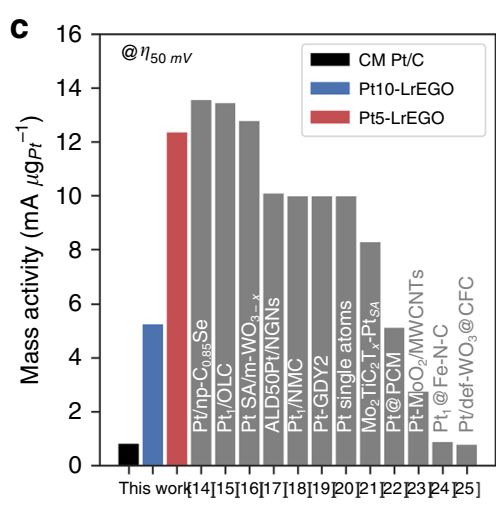

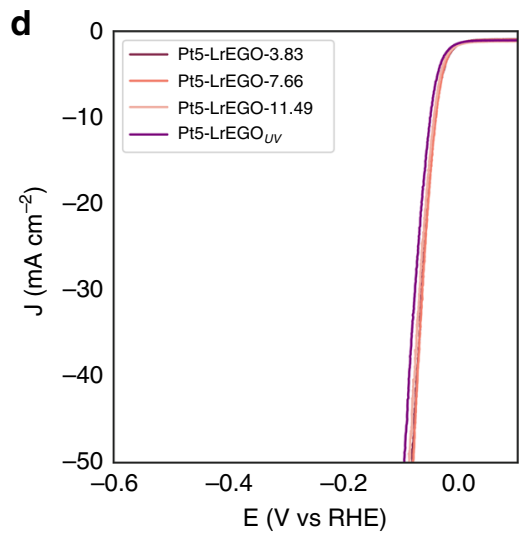

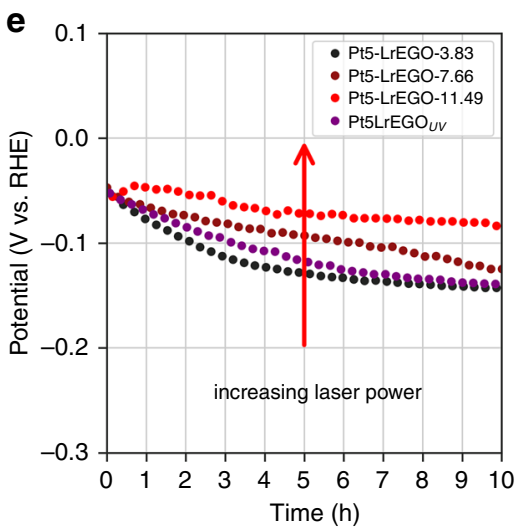

Fig. 6 Hydrogen evolution performance of the Pt-LrEGO samples. a LSVs (10 mV s${ }^{-1}$; after ohmic-drop correction) of the commercial Pt/C (20 $\left.w_{\mathrm{pt}_{\mathrm{p}}} \%\right)$ and the Pt-LrEGO catalysts prepared by laser irradiation $\left(1064 \mathrm{~nm} ; 7.66 \mathrm{~mJ} \mathrm{~cm}^{-2}\right.$ ) of the EGO films infused with Pt precursor solutions at different concentrations; the LSVs were recorded in $\mathrm{N}_{2}$-saturated $0.5 \mathrm{M} \mathrm{H}_{2} \mathrm{SO}_{4}$ at a rotation speed of $1600 \mathrm{rpm}$; the inset shows the overpotential of samples at a current density of $10 \mathrm{~mA} \mathrm{~cm}^{-2}$. b Tafel slopes of the commercial Pt/C and Pt10-LrEGO, Pt5-LrEGO, and Pt1-LrEGO. c Mass activities of the commercial Pt/C, Pt-LrEGO, and the literature reported Pt-based SACs at an overpotential $50 \mathrm{mV}$. The bibliographic information of the reference numbers can be found in Supplementary Table S6 in Supporting information. $\mathbf{d}$ LSV of Pt5-LrEGO samples obtained by $1064 \mathrm{~nm}$ laser irradiation at various laser fluences. e Chronopotentiometry of Pt-LrEGO samples treated at various laser fluences at a current density of $10 \mathrm{~mA} \mathrm{~cm}-2$

particles in Pt5-LrEGO ${ }_{\mathrm{UV}}$, Pt5-LrEGO-3.83, and Pt5LrEGO-7.66 (Supplementary Fig. S12c-h). The growth of the Pt active species might be ascribed to the migration of Pt atoms during the reaction because of the relatively weak atom-substrate interaction.

In addition, the emergence of $\mathrm{H}_{2}$ bubbles involved in the HER process can potentially cause damage to the basal plane of graphene ${ }^{50}$. The conductive pathways of 3Dinterconnected graphene substrates will be destroyed after long-term testing, thus leading to the degradation of electrocatalytic activity in HER. Therefore, graphene substrate with less structural defects and better mechanical property is expected to exhibit superior stability during the HER test. According to the Raman results mentioned above, the resultant LrEGO shows fewer structural defects at higher laser fluences. Therefore, LrEGO obtained at a higher fluence can bear the hydrogen evolution perturbation well. This is in good coincidence with the stability test mentioned above.
In addition, high-temperature treatment $(>1173 \mathrm{~K})$ can facilitate the stability of single atoms through a strong atom-substrate interaction, which potentially inhibits the migration and detachment of anchored atoms during HER ${ }^{16}$.

\section{Conclusions}

In summary, for the first time, we have developed a rapid, versatile, solid-phase laser manufacturing technique for synthesizing graphene-supported Pt SACs. In this approach, the sublimation of the precursor solution contains metal anionic complex $\left(\mathrm{PtCl}_{6}{ }^{2-}\right)$ promotes the "isolated dispersion" of $\mathrm{H}_{2} \mathrm{PtCl}_{6}$ precursor on the EGO substrate and prevents the localized aggregation by minimizing the electrostatic interaction between EGO and the metal ions. The pronounced photothermal effect of the 1064-nm laser irradiation in combination with fast scanning enables a high processing temperature (up to $1692.2 \mathrm{~K})$ with a short heating time $(\sim 1 \mathrm{~ms})$ and a rapid 
quenching time of $\sim 108 \mathrm{~ms}$, which not only guarantee the through-thickness thermal reduction of both EGO and Pt ions but also prevents the migration of metal atoms. We also demonstrated that the Pt-LrEGO exhibits promising activity and high Pt utilization in HER with an overpotential of $42.3 \mathrm{mV}$ at $10 \mathrm{~mA} / \mathrm{cm}^{2}$ and mass activity of $12.36 \mathrm{~mA} / \mu \mathrm{g}_{\mathrm{Pt}}$ at $50 \mathrm{mV}$. This laser-enabled solid-phase method potentially opens up a robust and versatile avenue for the production of a variety of single atoms on different substrates. Furthermore, this fast laser fabrication technique holds a promising potential in continuous roll-toroll processing and could be applied in high-throughput catalyst synthesis and screening.

\section{Experimental section Materials}

Electrochemical graphene oxide (EGO) was prepared using the previously reported two-step electrochemical intercalation and oxidation method ${ }^{31}$. Chloroplatinic acid hydrate $\left(\mathrm{H}_{2} \mathrm{PtCl}_{6} \times \mathrm{xH}_{2} \mathrm{O}\right.$, 99.995\%), platinum(IV) chloride $\left(\mathrm{PtCl}_{4}, 99.99 \%\right)$, Nafion (5\% in lower aliphatic alcohols and water) were purchased from Sigma-Aldrich and used as received. Platinum $\left(1000 \mu \mathrm{g} \mathrm{ml}^{-1}\right)$ ICP standard solution was purchased from SPEX CertiPrep. All chemicals were used as received without further purification, except dilution using deionized water $(15 \mathrm{M} \Omega \mathrm{cm})$.

\section{Preparation of LrEGO composites film}

To prepare the EGO film, $4 \mathrm{~mL}$ of $1 \mathrm{mg} \mathrm{mL}^{-1}$ EGO water dispersion was vacuum filtered through a PTFE filtration membrane $(0.2-\mu \mathrm{m}$ pore size, Millipore), the dimension of the EGO film was $3.2 \mathrm{~cm}$ in diameter and $1.5 \pm 0.2 \mu \mathrm{m}$ in thickness. In the case of Pt-EGO film, $1 \mathrm{~mL}$ metal precursor solution $\left(\mathrm{H}_{2} \mathrm{PtCl}_{6 /} \mathrm{PtCl}_{4}\right.$, $0.1-10 \mathrm{mmol} \mathrm{L}^{-1}$ ) was vacuum filtered through the EGO membrane. The EGO or Pt-EGO films were then immersed in liquid nitrogen and freeze-dried to reduce localized precipitation of metal salts. The dried GO and Pt-EGO films were subjected to direct laser beam patterning in constant Ar flow (Supplementary Fig. S1). Two laser sources were used to study the temperaturedependent SAC synthesis and the electrolysis stability: an infrared laser system of $1064 \mathrm{~nm}$ central wavelength (IPG Photonics, USA), $5 \mathrm{~ns}$ pulse width, $30 \mathrm{kHz}$ repetition rate, and average power $3-10 \mathrm{~W}$ was focused into the EGO film with a spot size of $\sim 1 \mathrm{~mm}$. an ultraviolet laser system $(355 \mathrm{~nm}, 10$ ps pulse duration, EdgeWave $\mathrm{GmbH}$, Germany) with pulse energy up to $54.4 \mu \mathrm{J}$, a repetition rate of $404 \mathrm{kHz}$, a spot size of $\sim 1 \mathrm{~mm}$. A raster scanner (Fig. 1d) with a line spacing of $0.5 \mathrm{~mm}$, which equals to the radius of the laser spot, was scanned across the PtEGO film in a parallel manner with a pattern dimension of $10 \mathrm{~mm} \times 10 \mathrm{~mm}$. The Galvo head scanning speed of both lasers was set to be $1000 \mathrm{~mm} / \mathrm{s}$.
The Pt-LrEGO were peeled off from the filtration membrane, the collected film samples were then sonicated in water, repeatedly washed (in deionized water, three times), and finally freeze-dried to obtain the catalyst powder for further electrochemical experiments.

\section{Materials characterization}

The LrEGO films after laser irradiation were characterized using scanning electron microscopy (SEM) performed on a field emission Zeiss Ultra 55 SEM operating at $3 \mathrm{kV}$ in lens mode. The high-resolution scanning transmission electron microscopy (HR-STEM) images and energy-dispersive X-ray spectroscopy (EDS) of Pt-LrEGO were captured by a $\mathrm{C}_{\mathrm{s}}$ corrected FEI Titan G2 80-200 S/TEM ChemiSTEM operating at $200 \mathrm{kV}$ equipped with a high-efficiency Super-X EDS detector system. To prepare TEM samples, the samples were dispersed and sonicated in ethanol as a dilution solution, then drop-casted onto the copper grid with a lacy carbon film. Raman spectroscopy was conducted using a Renishaw InVia Raman spectrometer, with a laser wavelength of $633 \mathrm{~nm}$ and a spot size of $2 \mu \mathrm{m}$; Raman spectra were analyzed using Wire 4.2 software, with Lorentzian function fitting after baseline subtraction. The peak intensity ratio of Raman D band to $\mathrm{G}$ band $\left(I_{\mathrm{D}} / I_{\mathrm{G}}\right)$ for $\mathrm{EGO}$ and rEGO was obtained from the fitted peak intensities. The full width at half-maximum (FWHM; $\Gamma$ ) of D and G bands was directly obtained from the curve fitting results. X-ray diffraction (XRD) were performed using a Proto AXRD benchtop powder X-ray diffractometer (XRD) with a $\mathrm{Cu}$ anode $(\lambda=1.5406 \AA)$ operating at $30 \mathrm{kV}$. X-ray photoelectron spectroscopy (XPS) was performed with a SPECS NAPXPS system; XPS spectra curve fittings were accomplished by CasaXPS software. The time-dependent temperature profile of the samples under laser irradiation was recorded using a FLIR T650sc infrared camera with a measurement range up to $2000^{\circ} \mathrm{C}$. The calibration of the infrared camera was conducted by the following steps ${ }^{51}$ : an EGO film was adhered on a $3 \mathrm{M}$ black electrical insulation tape and attached to a hotplate (Fisherbrand Isotemp, Fisher Scientific). The temperature was measured by a thermocouple (Model 88106-IEC, Omega) and controlled by the hotplate. The emissivity was calibrated through the infrared camera until the IR temperature is in accordance with the thermocouple measurement. The temporal temperature profile was recorded by averaging five individual points in each measurement. Four-point probe resistance measurements of the GO and LrEGO films were carried out using a four-point probe system (Janedel Engineering Ltd., Linslade, UK) equipped with a Keithley $2182 \mathrm{~A}$ nanovoltmeter and a Keithley 6220 current source (Keithley Instruments, Cleveland, OH, USA). To determine the mass of metal content within the Pt-LrEGO catalysts, an inductively coupled plasma-optical emission spectrometry (ICP-OES) was conducted by using a Analytikjena PlasmaQuant 9000 
Elite system. All samples were weighed and digested in aqua regia for two days, the solution was then diluted and filtered with a $0.2-\mu \mathrm{m}$ pore size Whatman syringe filter (Fisher Scientific, UK).

\section{Finite element (FE) simulation}

The time-dependent FE simulation of the pulsed-laser patterning process on the EGO film was performed using COMSOL modeling to acquire a better understanding of the effects of laser fluence and NOPs on the temporal temperature elevation. An EGO film with $2 \mu \mathrm{m}$ thick and dimensions of $1 \mathrm{~cm}$ by $1 \mathrm{~cm}$ was modeled as a candidate material, where its temperature-dependent material properties are acquired by taking typical values from literature (Supplementary Table S5). The pulsed laser with a Gaussian power density distribution is modeled as a heat flux on the surface of the EGO film. The heat transfer within the materials is governed by the COMSOL built-in general heat equation:

$$
\rho C_{p} \frac{\partial T}{\partial t}+\rho C_{p} u \cdot \nabla T+\nabla \cdot(-k \nabla T)=Q
$$

where the first, second, and third terms on the left-hand side are the time derivative of thermal energy per unit volume, convection energy, and conduction energy, respectively. $T$ is the local temperature, $t$ is the time span, $Q$ is the converted energy from the laser beam to the material through photothermal conversion. $\rho, C_{p}$, and $k$ are mass density, specific heat capacitance, and thermal conductivity, respectively. The upper boundary of the film was set to be exposed to the boundary conditions of heat flux, natural convection, and irradiative convection. The lower boundary was subjected to an insulated boundary.

The pulsed-laser heat source with a Gaussian profile is shown in the following equation ${ }^{38,48}$ :

$$
Q=A \cdot P \cdot \operatorname{Beam}(x, y, t) \cdot \operatorname{Pulsetrain}(t)
$$

where $A$ is the absorptivity at a given incident radiation wavelength, $P$ is the laser output power density, Beam $(x, y, t)$ describes the Gaussian laser beam shape and its displacement, and Pulsetrain $(t)$ describes the laser timedependent pulse parameter. The Gaussian power density distribution of the laser beam was described as the following expression:

$$
\operatorname{Beam}(x, y, t)=\exp \left\{-\frac{\left[x-\left(x_{0}-v_{x} t\right)\right]^{2}}{2 \phi^{2}}+\frac{y^{2}}{2 \phi^{2}}\right\}
$$

where $\mathrm{x}$ and $\mathrm{y}$ are the coordinates of the laser beam center, $\mathrm{v}_{\mathrm{x}}$ is the beam travel velocity along the $x$ direction, $t$ is the time span, and $\phi$ is the standard deviation of the Gaussian laser beam. The thermal and optical properties of EGO used in the calculation are listed in Supplementary Table S5.

\section{Electrochemical experiments}

All electrochemical experiments were performed in a conventional three-electrode system at a VersaSTAT4 Potentiostat (AMETEK, USA) and a $636 \mathrm{~A}$ electrode rotator system (AMETEK, USA) with an $\mathrm{Ag} / \mathrm{AgCl}$ (saturated $\mathrm{KCl}$ solution) electrode as the reference electrode, a coiled platinum wire as the counter electrode, and a glassy carbon rotating disk electrode (RDE, Pine research) with a diameter of $5 \mathrm{~mm}\left(0.196 \mathrm{~cm}^{2}\right)$ as the working electrode. All potential was converted to the RHE by using the following equation: $E_{\mathrm{RHE}}=E_{\mathrm{Ag} / \mathrm{AgCl}}+0.197+0.059 \mathrm{pH}$. The RDE was polished with a microfiber polishing cloth with $0.05-\mu \mathrm{m}$ alumina slurry until the mirror finish prior to all experiments. In all, $10 \mathrm{mg}$ of catalyst was mixed with deionized water, isopropanol (99\%, Aldrich), and Nafion (5\%, Aldrich) with a volume ratio of 9:10:1, respectively, to a final concentration of $5 \mathrm{mg} \mathrm{mL}^{-1}$. After ultrasonication in an ice bath for $1 \mathrm{~h}, 10 \mu \mathrm{L}$ of homogeneous ink was drop-casting on the RDE and dried under ambient, with a final loading of $50 \mu \mathrm{g}\left(\sim 0.255 \mathrm{mg} \mathrm{cm}^{-2}\right)$. The Ohmic losses within the system were compensated by applying $I R$-correction. The uncompensated system resistance was determined by electrochemical impedance spectroscopy (EIS) at the open-circuit potential. The EIS was measured in a range of $100 \mathrm{kHz}$ to $1 \mathrm{~Hz}$, with a perturbation of $10 \mathrm{mV}$. The system resistance was then determined at the $\mathrm{x}$-intercept of the Nyquist plot. The Nyquist plot during HER was based on EIS measurements at an overpotential of $30 \mathrm{mV}$ in a frequency range of $10^{-2}$ to $10^{6} \mathrm{~Hz}$ with $10 \mathrm{mV}$ sinusoidal perturbations in $0.5 \mathrm{M} \mathrm{H}_{2} \mathrm{SO}_{4}$. Linear sweep voltammetry with a scan rate of $10 \mathrm{mV} \mathrm{s}^{-1}$ was conducted in $\mathrm{N}_{2}$ saturated $0.5 \mathrm{M} \mathrm{H}_{2} \mathrm{SO}_{4}$ at a rotating speed of $1600 \mathrm{rpm}$. To obtain the current density, the current was normalized by the geometric electrode area, which is $\sim 0.196 \mathrm{~cm}^{2}$. Chronopotentiometry measurements (at $10 \mathrm{~mA} \mathrm{~cm}^{-2}$ ) were conducted to evaluate the long-term HER stability.

\footnotetext{
Acknowledgements

The work made use of the facilities at The University of Manchester Electron Microscopy Centre. The authors acknowledge the use of the Department of Materials X-ray Diffraction Suite at The University of Manchester and are grateful for the technical support and advice by Dr. John E. Warren.

\section{Author details}

${ }^{1}$ Department of Materials, School of Natural Sciences, The University of Manchester, Manchester M13 9PL, UK. ${ }^{2}$ National Graphene Institute, The University of Manchester, Manchester M13 9PL, UK. ${ }^{3}$ Laser Processing Research Centre, Department of Mechanical, Aerospace and Civil Engineering, The University of Manchester, Manchester M13 9PL, UK
}

\section{Author contributions}

Y.P. and J.C. conceived the idea and designed the experiments in discussion with Z.L. and L.L. Y.P. and J.C. prepared the EGO film samples; Y.P. and Y.S. prepared the LrEGO and Pt-LrEGO samples; Y.P. carried out all the 
characterization and measurements and Finite Element modeling. J.C. helped with Raman spectra characterization and analysis, W.Y. helped with sample preparation; Y.P. wrote the paper with the help of all authors. All authors have approved the final version of the paper. The research was carried out under the direction and supervision of Z.L. and L.L.

\section{Conflict of interest}

The authors declare no competing interests.

Supplementary information The online version contains supplementary material available at https://doi.org/10.1038/s41377-021-00603-9.

Received: 22 January 2021 Revised: 25 June 2021 Accepted: 19 July 2021 Published online: 18 August 2021

\section{References}

1. $\mathrm{Pu}, \mathrm{Z}$. H. et al. Single-atom catalysts for electrochemical hydrogen evolution reaction: recent advances and future perspectives. Nano-Micro Lett. 12, 21 (2020).

2. Yang, M. \& Flytzani-Stephanopoulos, M. Design of single-atom metal catalysts on various supports for the low-temperature water-gas shift reaction. Catal. Today 298, 216-225 (2017).

3. Yan, $\mathrm{H}$. et al. Single-atom catalysts and their applications in organic chemistry. J. Mater. Chem. A 6, 8793-8814 (2018).

4. Liu, J. et al. High performance platinum single atom electrocatalyst for oxygen reduction reaction. Nat. Commun. 8, 15938 (2017).

5. Cheng, N. C. et al. Single-atom catalysts: from design to application. Electrochem. Energy Rev. 2, 539-573 (2019).

6. Liu, D. B. et al. Atomically dispersed platinum supported on curved carbon supports for efficient electrocatalytic hydrogen evolution. Nat. Energy 4, 512-518 (2019).

7. Chen, Y. X. et al. Fabrication, characterization, and stability of supported singleatom catalysts. Catal. Sci. Technol. 7, 4250-4258 (2017).

8. Wei, H. S. et al. $\mathrm{FeO}_{x}$-supported platinum single-atom and pseudo-single-atom catalysts for chemoselective hydrogenation of functionalized nitroarenes. Nat. Commun. 5, 5634 (2014).

9. Lin, L. L. et al. Low-temperature hydrogen production from water and methanol using Pt/a-MoC catalysts. Nature 544, 80-83 (2017).

10. Li, M. F. et al. Single-atom tailoring of platinum nanocatalysts for highperformance multifunctional electrocatalysis. Nat. Catal. 2, 495-503 (2019).

11. Fei, H. L. et al. Single atom electrocatalysts supported on graphene or graphene-like carbons. Chem. Soc. Rev. 48, 5207-5241 (2019).

12. Chen, Y. J. et al. Single-atom catalysts: synthetic strategies and electrochemical applications. Joule 2, 1242-1264 (2018).

13. Zhang, J. et al. Cation vacancy stabilization of single-atomic-site $\mathrm{Pt}_{1} / \mathrm{Ni}$ $(\mathrm{OH})_{x}$ catalyst for diboration of alkynes and alkenes. Nat. Commun. 9 , 1002 (2018).

14. Cheng, W. R. et al. Lattice-strained metal-organic-framework arrays for bifunctional oxygen electrocatalysis. Nat. Energy 4, 115-122 (2019).

15. Cheng, Y. et al. Atomically dispersed transition metals on carbon nanotubes with ultrahigh loading for selective electrochemical carbon dioxide reduction. Adv. Mater. 30, 1706287 (2018).

16. Yao, Y. G. et al. High temperature shockwave stabilized single atoms. Nat. Nanotechnol. 14, 851-857 (2019).

17. Qu, Y. T. et al. Thermal emitting strategy to synthesize atomically dispersed Pt metal sites from bulk Pt metal. J. Am. Chem. Soc. 141, 4505-4509 (2019).

18. Jiang, $\mathrm{K}$. et al. Isolated $\mathrm{Ni}$ single atoms in graphene nanosheets for highperformance $\mathrm{CO}_{2}$ reduction. Energy Environ. Sci. 11, 893-903 (2018).

19. Zhao, Y. et al. Integrated graphene systems by laser irradiation for advanced devices. Nano Today 12, 14-30 (2017).

20. Zeng, H. B. et al. Nanomaterials via laser ablation/irradiation in liquid: a review. Adv. Funct. Mater. 22, 1333-1353 (2012).

21. Zhang, D. S., Gökce, B. \& Barcikowski, S. Laser synthesis and processing of colloids: fundamentals and applications. Chem. Rev. 117, 3990-4103 (2017).

22. Gengler, R. Y. N. et al. Revealing the ultrafast process behind the photoreduction of graphene oxide. Nat. Commun. 4, 2560 (2013).
23. Moussa, S. et al. Laser assisted photocatalytic reduction of metal ions by graphene oxide. J. Mater. Chem. 21, 9608-9619 (2011).

24. Yin, X. P. et al. Engineering the coordination environment of single-atom platinum anchored on graphdiyne for optimizing electrocatalytic hydrogen evolution. Angew. Chem. Int. Ed. 57, 9382-9386 (2018).

25. Jin, Z. S. et al. On the conditions and mechanism of $\mathrm{PtO}_{2}$ formation in the photoinduced conversion of $\mathrm{H}_{2} \mathrm{PtCl}_{6}$. J. Photochem. Photobiol. A: Chem. 81, 177-182 (1994)

26. Xuyen, N. T. et al. Hydrolysis-induced immobilization of Pt(acac) $)_{2}$ on polyimide-based carbon nanofiber mat and formation of Pt nanoparticles. J. Mater. Chem. 19, 1283-1288 (2009).

27. Labinger, J. A. \& Bercaw, J. E. Understanding and exploiting $\mathrm{C}-\mathrm{H}$ bond activation. Nature 417, 507-514 (2002).

28. Sitko, R. et al. Adsorption of divalent metal ions from aqueous solutions using graphene oxide. Dalton Trans. 42, 5682-5689 (2013).

29. $\mathrm{Mi}$, X. et al. Preparation of graphene oxide aerogel and its adsorption for $\mathrm{Cu}^{2+}$ ions. Carbon 50, 4856-4864 (2012).

30. Lowde, D. R. et al. Characterization of electro-oxidation catalysts prepared by ion-exchange of platinum salts with surface oxide groups on carbon. J. Chem. Soc., Faraday Trans. 1: Phys. Chem. Condens. Phases 75, 2312-2324 (1979).

31. Cao, J. Y. et al. Two-step electrochemical intercalation and oxidation of graphite for the mass production of graphene oxide. J. Am. Chem. Soc. 139 17446-17456 (2017).

32. Eckmann, A. et al. Probing the nature of defects in graphene by Raman spectroscopy. Nano Lett. 12, 3925-3930 (2012).

33. Cançado, L. G. et al. Quantifying defects in graphene via Raman spectroscopy at different excitation energies. Nano Lett. 11, 3190-3196 (2011).

34. Lucchese, M. M. et al. Quantifying ion-induced defects and Raman relaxation length in graphene. Carbon 48, 1592-1597 (2010).

35. Ferrari, A. C. \& Basko, D. M. Raman spectroscopy as a versatile tool for studying the properties of graphene. Nat. Nanotechnol. 8, 235-246 (2013).

36. Peng, Y. D. et al. Laser assisted solution synthesis of high performance graphene supported electrocatalysts. Adv. Funct. Mater. 30, 2001756 (2020).

37. Guo, H. L. et al. Preparation of reduced graphene oxide by infrared irradiation induced photothermal reduction. Nanoscale 5, 9040-9048 (2013).

38. Chen, $X$. et al. Nanosecond photothermal effects in plasmonic nanostructures. ACS Nano 6, 2550-2557 (2012).

39. Schweizer, A. E. \& Kerr, G. T. Thermal decomposition of hexachloroplatinic acid. Inorg. Chem. 17, 2326-2327 (1978)

40. Acik, M. et al. The role of oxygen during thermal reduction of graphene oxide studied by infrared absorption spectroscopy. J. Phys. Chem. C. 115, 19761-19781 (2011)

41. Bagri, A. et al. Structural evolution during the reduction of chemically derived graphene oxide. Nat. Chem. 2, 581-587 (2010).

42. Arul, R. et al. The mechanism of direct laser writing of graphene features into graphene oxide films involves photoreduction and thermally assisted structural rearrangement. Carbon 99, 423-431 (2016).

43. Sokolov, D. A., Shepperd, K. R. \& Orlando, T. M. Formation of graphene features from direct laser-induced reduction of graphite oxide. J. Phys. Chem. Lett. 1, 2633-2636 (2010).

44. Schmidt, $H$. et al. Ultraviolet laser ablation of polymers: spot size, pulse duration, and plume attenuation effects explained. J. Appl. Phys. 83, 5458-5468 (1998).

45. Lin, J. et al. Laser-induced porous graphene films from commercial polymers Nat. Commun. 5, 5714 (2014).

46. Luong, D. X. et al. Gram-scale bottom-up flash graphene synthesis. Nature 577, 647-651 (2020).

47. Renteria, J. D. et al. Strongly anisotropic thermal conductivity of free-standing reduced graphene oxide films annealed at high temperature. Adv. Funct. Mater. 25, 4664-4672 (2015).

48. Zhou, Y. et al. Microstructuring of graphene oxide nanosheets using direct laser writing. Adv. Mater. 22, 67-71 (2010).

49. Huang, Z. P. et al. Cobalt phosphide nanorods as an efficient electrocatalyst for the hydrogen evolution reaction. Nano Energy 9, 373-382 (2014).

50. Ferrari, A. G. M., Brownson, D. A. C. \& Banks, C. E. Investigating the integrity of graphene towards the electrochemical hydrogen evolution reaction (HER). Sci. Rep. 9, 15961 (2019).

51. Xiao, L. et al. Fast adaptive thermal camouflage based on flexible $\mathrm{VO}_{2} / \mathrm{Gra}$ phene/CNT thin films. Nano Lett. 15, 8365-8370 (2015). 